\title{
THE TORSION OF THE GROUP OF HOMEOMORPHISMS OF POWERS OF THE LONG LINE
}

\author{
SATYA DEO and DAVID GAULD \\ (Received 4 October 1999; revised 18 October 2000)
}

Communicated by J. A. Hillman

\begin{abstract}
By blending techniques from set theory and algebraic topology we investigate the order of any homeomorphism of the $n$th power of the long ray or long line $\mathbb{L}$ having finite order, finding all possible orders when $n=1,2,3$ or 4 in the first case and when $n=1$ or 2 in the second. We also show that all finite powers of $\mathbb{L}$ are acyclic with respect to Alexander-Spanier cohomology.
\end{abstract}

2000 Mathematics subject classification: primary 55M35, 57N65, 57N80, 57S17, $03 \mathrm{E} 75$.

Keywords and phrases: long line, long ray, Alexander-Spanier cohomology, torsion of homeomorphisms.

\section{Introduction}

Topologists have adapted two powerful tools from other branches of mathematics to assist them in solving topological problems: algebra and set theory. Each has made major contributions but in distinct areas of topology. Algebraic topology has been very effective in the context of compact spaces, essentially requiring finiteness. Set theory has been effective in dealing with the large infinite. In the context of topological manifolds, algebraic topology has been invaluable in the study of compact manifolds while set theory has been of most use in the study of non-metrisable manifolds. Unfortunately it has been unusual for the two to be combined. In this paper we discuss one way in which these techniques can work together and as a result solve a problem in the theory of non-metrisable manifolds.

Both authors were supported in part by a Marsden Fund Award, UOA611, from the Royal Society of New Zealand. The second author also received partial support from the DRS Programme of the Department of Mathematics, R. D. University, Jabalpur.

(C) 2001 Australian Mathematical Society 0263-6115/2001 \$A2.00+0.00 
We denote by $\omega_{1}$ the set of countable ordinals and by $\mathbb{L}$ the long line, which is obtained by inserting an open interval between each countable ordinal and its successor to obtain the closed long ray $\mathbb{L}_{+}$and then joining two copies of $\mathbb{L}_{+}$(which we denote by $\mathbb{L}_{+}$and $\mathbb{L}_{-}$) by identifying the ordinals $0 \in \mathbb{L}_{+}$and $0 \in \mathbb{L}_{-}$. We denote the open long ray, which is $\mathbb{L}_{+}$with 0 removed, by $\mathbb{L}_{0}$.

The primary goal of this paper is to determine the torsion of the group of homeomorphisms of low powers of the long line and long ray. Our main result shows that the only torsion in these groups is torsion corresponding to permutation of the coordinates and 2-torsion. The computation involves a blend of notions of the set theory of the countable ordinals and algebraic topology, particularly the P. A. Smith theorems. Details appear in Section 3.

In order to apply the P. A. Smith theorems we find it necessary to calculate the Alexander-Spanier cohomology of powers of the long line. Of course it is trivial to show that in singular cohomology theory all powers of the long line are acyclic. We find in Section 3 that in Alexander-Spanier cohomology theory the powers are also acyclic. Although we will work with coefficients in an $R$-module $G$, we will suppress this in the notation.

We refer the reader to [6, Section 6.4] for a discussion of the Alexander-Spanier cohomology groups $\bar{H}^{q}(X)$.

Proofs of the following results may be found in many books on set theory, for example [5, page 78 and 80$]$.

PROPOSITION 1.1. The intersection of a countable collection of closed, unbounded subsets of $\omega_{1}$ is again closed and unbounded.

PROPOSITION 1.2 (Pressing Down Lemma). Let $S \subset \omega_{1}$ be a stationary set and $f: S \rightarrow \omega_{1}$ be a regressive function. Then there is $\alpha \in \omega_{1}$ so that $f^{-1}(\alpha)$ is stationary.

A function $f: A \rightarrow \omega_{1}$ is regressive if for each $\alpha \in A \backslash\{0\} \subset \omega_{1}$ we have $f(\alpha)<\alpha$. A set $S \subset \omega_{1}$ is stationary provided that it meets every closed, unbounded subset of $\omega_{1}$.

\section{The cohomology of powers of the long line}

Whenever $x$ is a point in the finite product of sets we denote by $x_{i}$ the $i$ th coordinate of $x$. If $\alpha \in \mathbb{L}$ let $\tilde{\alpha}=(\alpha, \ldots, \alpha) \in \mathbb{L}^{m}$. For $t \in \mathbb{R}^{l}$ and $r>0$ set

$$
C[t ; r]=\left\{s \in \mathbb{R}^{k} \mid \forall j=1, \ldots, l, t_{j}-r \leq s_{j} \leq t_{j}+r\right\} .
$$


For $x, y \in \mathbb{L}^{m}$ set

$$
D[x, y]=\left\{z \in \mathbb{L}^{m} \mid \forall j=1, \ldots, m, \min \left\{x_{j}, y_{j}\right\} \leq z_{j} \leq \max \left\{x_{j}, y_{j}\right\}\right\} .
$$

LEMMA 2.1. Let $l, m \geq 0$ be integers and let $\mathscr{U}$ be a collection of open subsets of $\mathbb{R}^{l} \times \mathbb{L}_{+}^{m}$ which covers $[0,1]^{l} \times \mathbb{L}_{+}^{m}$. Then there are $\alpha \in \mathbb{L}_{+}$and a cover $\left\{J_{1}, \ldots, J_{p}\right\}$ of $[0,1]$ by open intervals such that for each $s, t \in[0,1]^{l}$ and each $x, y \in \mathbb{L}_{+}^{m}$ if for each $j=1, \ldots$, there is $i_{j}$ with $s_{j}, t_{j} \in J_{i_{j}}$ and $x_{j}, y_{j} \geq \alpha$ for each $j=1, \ldots, m$ then there is $U \in \mathscr{U}$ with $(s, x),(t, y) \in U$.

PROOF. For each $t \in[0,1]^{l}$ and each limit ordinal $\lambda \in \mathbb{L}_{+}$we have $(t, \tilde{\lambda}) \in$ $[0,1\}^{l} \times \mathbb{L}_{+}^{m}$ so there is $U \in \mathscr{U}$ with $(t, \tilde{\lambda}) \in U$. Thus there are an integer $n>0$ and an ordinal $f(\lambda)<\lambda$ so that $C[t ; 1 / n] \times D[\widetilde{f(\lambda)}, \tilde{\lambda}] \subset U$.

By the Pressing Down Lemma there is an ordinal $\alpha_{t}$ such that $f^{-1}\left(\alpha_{t}\right)$ is uncountable, hence there are an unbounded set $S_{t} \subset f^{-1}\left(\alpha_{t}\right)$ and an integer $n_{t}>0$ such that for each $\lambda \in S_{t}$ there is $U \in \mathscr{U}$ with $C\left[t ; 1 / n_{t}\right] \times D\left[\tilde{\alpha}_{t}, \tilde{\lambda}\right] \subset U$.

By compactness finitely many interiors of the hypercubes $\left\{C\left[t ; 1 / n_{t}\right] \mid t \in[0,1]^{l}\right\}$ cover $[0,1]^{l}$; say $\left\{C\left[t_{(i)} ; 1 / n_{i}\right] \mid i=1, \ldots q\right\}$, where we abbreviate $n_{t_{(i)}}$ to $n_{i}$; we similarly abbreviate $\alpha_{t_{(i)}}$ and $S_{t_{(i)}}$. Let $\alpha=\max \left\{\alpha_{i} \mid i=1, \ldots, q\right\}$. From the hypercubes $\left\{C\left[t_{(i)} ; 1 / n_{i}\right] \mid i=1, \ldots q\right\}$ we may construct open intervals $\left\{J_{1}, \ldots, J_{p}\right\}$ covering $[0,1]$ such that each of the sets $\prod_{j=1}^{l} J_{i j}$ lies in some $C\left[t_{(i)} ; 1 / n_{i}\right]$.

Suppose that $s, t \in[0,1]^{l}$ and $x, y \in \mathbb{L}_{+}^{m}$ are such that for each $j=1, \ldots, l$ there is $i_{j}$ with $s_{j}, t_{j} \in J_{i_{j}}$ and $x_{j}, y_{j} \geq \alpha$ for each $j=1, \ldots, m$. Then $s, t \in C\left[t_{(i)} ; 1 / n_{i}\right]$ for some $i$. Choose any $\lambda \in S_{i}$ such that $x_{j}, y_{j} \leq \lambda$ for each $j$; then $x, y \in D\left[\tilde{\alpha}_{i}, \bar{\lambda}\right]$. Choose $U \in \mathscr{U}$ such that $C\left[t_{(i)} ; 1 / n_{i}\right] \times D\left[\tilde{\alpha}_{i}, \tilde{\lambda}\right] \subset U$. Then $(s, x),(t, y) \in U$.

Proposition 2.2. $\tilde{\bar{H}}^{q}\left(\mathbb{L}_{+}\right)=0$ for each $q \geq 0$.

PROOF. Since $\mathbb{L}_{+}$is a connected space, by $[6$, Corollary 6.4.7] it follows that $\tilde{H}^{0}\left(\mathbb{L}_{+}\right)$is trivial, so we will assume that $q \geq 1$.

Let $[\varphi] \in \bar{H}^{q}\left(\mathbb{L}_{+}\right)=\bar{H}^{q}\left(\mathbb{L}_{+}\right)$be an arbitrary cohomology class, where $\varphi$ : $\mathbb{L}_{+}^{q+1} \rightarrow G$ is a $q$-cocycle. Then $\delta \varphi$ is locally zero on $\mathbb{L}_{+}$so there is an open covering $\mathscr{U}$ of $\mathbb{L}_{+}$such that whenever $x_{0}, \ldots, x_{q+1} \in U \in \mathscr{U}$ then $\delta \varphi\left(x_{0}, \ldots, x_{q+1}\right)=0$. By Lemma 2.1 there is $\alpha \in \mathbb{L}_{+}$such that for any $\beta \in\left(\alpha, \omega_{1}\right)$ the interval $[\alpha, \beta]$ is contained in some member of $\mathscr{U}$. Given any $x_{0}, \ldots, x_{q+1} \in\left[\alpha, \omega_{1}\right)$ we can choose $\beta$ so that $x_{0}, \ldots, x_{q+1}<\beta$; so $x_{0}, \ldots, x_{q+1} \in U \in \mathscr{U}$ and hence $\delta \varphi\left(x_{0}, \ldots, x_{q+1}\right)=0$. Thus by [6, Lemma 6.4.1] there is $\psi \in C^{q-1}\left(\mathbb{L}_{+}\right)$such that on $\left[\alpha, \omega_{1}\right)^{q+1}$ we have $\delta \psi=\varphi$. It follows that $[\varphi] \mid\left(\alpha, \omega_{1}\right)=0$.

Let $V=[0, \alpha+1)$ and $W=\left(\alpha, \omega_{1}\right)$. As $V \cup W=\mathbb{L}_{+}$and $V$ and $V \cap$ $W$ are contractible, restriction induces an isomorphism $\tilde{H}^{q}\left(\mathbb{L}_{+}\right) \approx \tilde{\tilde{H}}^{q}(W)$ by the Mayer-Vietoris sequence for reduced Alexander-Spanier cohomology for the triple $\left(\mathbb{L}_{+} ; V, W\right)$. Since $[\varphi] \mid W=0$ we have $[\varphi]=0$. Hence $\mathbb{L}_{+}$is acyclic. 
COROLLARY 2.3. The Alexander-Spanier cohomology of the long line is given by:

$$
\bar{H}^{q}(\mathbb{L})=\left\{\begin{array}{lll}
G & \text { if } & q=0 \\
0 & \text { if } & q>0 .
\end{array}\right.
$$

PROOF. Apply a Mayer-Vietoris argument to $\left(\mathbb{L} ; \mathbb{L}_{+}, \mathbb{L}_{-}\right)$.

PROPOSITION 2.4. For each $n \geq 1$ and each $q \geq 0$ the reduced Alexander-Spanier cohomology groups $\tilde{H}^{q}\left(\mathbb{L}_{+}^{n}\right)$ are all trivial.

ProOF. We use induction on $n$, the case $n=1$ having been shown in Proposition 2.2. Assume the result true for powers less than $n$.

Let $[\varphi] \in \tilde{\tilde{H}}^{q}\left(\mathbb{R}_{+}^{n}\right)$, where $\varphi$ is a cocycle. Then there is an open cover $\mathscr{U}$ of $\mathbb{L}_{+}^{n}$ such that $\delta \varphi$ is locally zero on $\mathscr{U}^{q+2}$. Hence by Lemma 2.1 there is $\alpha<\omega_{1}$ such that $\delta \varphi$ is identically zero on $\left(\left[\alpha, \omega_{1}\right)^{n}\right)^{q+2}$. Hence by $[6$, Lemma 6.4.1] there is $\psi \in C^{q-1}\left(\left[\alpha, \omega_{1}\right)^{n}\right)$ such that $\delta \psi=\varphi \mid\left[\alpha, \omega_{1}\right)^{n}$. Thus $[\varphi] \mid\left[\alpha, \omega_{1}\right)^{n}=0$.

We now prove by induction on $m$ that $[\varphi] \mid\left[0, \omega_{1}\right)^{m} \times\left[\alpha, \omega_{1}\right)^{n-m}=0$, having already begun the induction at $m=0$. Suppose that $[\varphi] \mid\left[0, \omega_{1}\right)^{m-1} \times\left[\alpha, \omega_{1}\right)^{n-m+1}=0$. Note that

$$
\begin{aligned}
& {\left[0, \omega_{1}\right)^{m} \times\left[\alpha, \omega_{1}\right)^{n-m}} \\
& \quad=\left[0, \omega_{1}\right)^{m-1} \times\left([0, \alpha] \cup\left[\alpha, \omega_{1}\right)\right) \times\left[\alpha, \omega_{1}\right)^{n-m} \\
& =\left(\left[0, \omega_{1}\right)^{m-1} \times[0, \alpha] \times\left[\alpha, \omega_{1}\right)^{n-m}\right) \cup\left(\left[0, \omega_{1}\right)^{m-1} \times\left[\alpha, \omega_{1}\right)^{n-m+1}\right) .
\end{aligned}
$$

Thus we have expressed $\left[0, \omega_{1}\right)^{m} \times\left[\alpha, \omega_{1}\right)^{n-m}$ as a union of two closed subsets. By inductive hypotheses with respect to $n,[\varphi] \mid\left[0, \omega_{1}\right)^{m-1} \times[0, \alpha] \times\left[\alpha, \omega_{1}\right)^{n-m}=0$ because the compact interval $[0, \alpha]$ is contractible, and by inductive hypothesis with respect to $m$ we have $[\varphi] \mid\left[0, \omega_{1}\right)^{m-1} \times\left[\alpha, \omega_{1}\right)^{n-m+1}=0$. Furthermore the intersection of these two closed subsets is $\left[0, \omega_{1}\right)^{m-1} \times\{\alpha\} \times\left[\alpha, \omega_{1}\right)^{n-m}$, which is homeomorphic to $\left[0, \omega_{1}\right)^{m-1} \times\left[\alpha, \omega_{1}\right)^{n-m}$ so again by inductive hypothesis is acyclic. Thus by the reduced Mayer-Vietoris sequence for these two closed sets we may conclude that $[\varphi] \mid\left[0, \omega_{1}\right)^{m} \times\left[\alpha, \omega_{1}\right)^{n-m}=0$.

Taking $m=n$ in the statement above, we conclude that $[\varphi]=0$. Thus $\tilde{H}^{q}\left(\mathbb{L}_{+}^{n}\right)=0$.

THEOREM 2.5. The Alexander-Spanier cohomology of powers of the long line is given by:

$$
\bar{H}^{q}\left(\mathbb{L}^{n}\right)=\left\{\begin{array}{lll}
G & \text { if } & q=0 \\
0 & \text { if } & q>0
\end{array}\right.
$$


PROOF. The product $\mathbb{L}^{0} \times \mathbb{L}_{+}^{n}$ is acyclic for all $n$ by Proposition 2.4. Inductive use of the Mayer-Vietoris sequence applied to the triples $\left(\mathbb{L}^{m} \times \mathbb{L}_{+}^{n-m} ; \mathbb{L}^{m-1} \times \mathbb{L}_{+}^{n-m+1}, \mathbb{L}^{m-1} \times\right.$ $\left.\mathbb{L}_{-} \times \mathbb{L}_{+}^{n-m}\right)$ now gives the result.

\section{Torsion of the group of homeomorphisms of powers of the long line}

In this section we study the group of homeomorphisms, $\mathscr{H}\left(L^{n}\right)$, of the space $L^{n}$, where $L$ is either $\mathbb{L}$ or $\mathbb{L}_{+}$. For each $\alpha \in \omega_{1}$ we denote by $S_{\alpha}=\left\{x \in \mathbb{L}^{n}|| x \mid \leq \alpha\right\}$ the 'square' of sides $2 \alpha$.

Let

$$
\begin{aligned}
|x| & =\max \left\{\left|x_{i}\right| \mid i=1, \ldots n\right\}, \\
\hat{x} & =\left\{i \in\{1, \ldots, n\}|| x_{i}|=| x \mid\right\}, \\
\check{x} & =\{1, \ldots, n\}-\hat{x}
\end{aligned}
$$

and

$$
\left.\|x\|=\max \left\{\left|x_{i}\right| \mid i \in \check{x}\right\} \quad \text { (with }\|x\|=0 \text { if } \hat{x}=\{1, \ldots, n\}\right) .
$$

Although Theorem 3.2 and Theorem 3.3 are subsumed in later results of this section we present them independently because the proofs which we present here show a strong interplay between algebraic topology and set theory. In order to apply Smith theory for locally compact spaces, we need to show that the space is of finite cohomological dimension [1]. However, that follows very quickly from the next proposition.

PROPOSITION 3.1. The cohomological dimension of the long line, $\operatorname{dim}_{\mathbb{Z}}(\mathbb{L})$ is 1 .

PROOF. As a 1-manifold, $\mathbb{L}$ is locally compact. The cohomological dimension of a locally compact space is determined locally and, as the local cohomological dimension of $\mathbb{L}$ is 1 , so is the (global) cohomological dimension.

THEOREM 3.2. Let $L$ denote either $\mathbb{L}_{+}$or $\mathbb{L}_{0}$. Then the group $\mathscr{H}(L)$ has no torsion.

PROOF. It is enough to show that $\mathscr{H}(L)$ has no elements of prime order. Suppose to the contrary that there is a homeomorphism $h: L \rightarrow L$ of prime order $p$. Let $G=[h]$ be the cyclic group of order $p$ generated by $h$, and $L^{h}=\{x \in L \mid h(x)=x\}$ be the fixed point set of $h$. Evidently $L^{h}=L^{G}$, the fixed point set of the group $G$.

The space $L$ is a locally compact Hausdorff space which, by Proposition 2.2, is acyclic $(\bmod p$ ) with respect to Alexander-Spanier cohomology, and $G$ is a group of order $p$. Hence by the Smith theorem, [3], the fixed point set $L^{G}$ must also be acyclic $(\bmod p)$. Hence $L^{G}$ must be connected by [6, Corollary 6.4.7]. We also know from the proof of $\left[4\right.$, Lemma 2] that $L^{h}$ must contain an unbounded subset of $\omega_{1}$. It follows that $L^{h}=L^{G}=L$, that is, that $h$ is the identity, a contradiction. 
THEOREM 3.3. The group $\mathscr{H}(\mathbb{L})$ has only 2-torsion, that is, any nontrivial element of finite order must be of order 2.

PROOF. Let $h \in \mathscr{H}(\mathbb{Q})$ be a nontrivial homeomorphism of finite prime power order, say $h^{p^{k}}=e$, the identity. It suffices to show that $p^{k}=2$, that is, that $p=2$ and $k=1$.

We remark that the Smith theorems are also valid for prime-power order groups, for any prime $p$. It follows from Corollary 2.3 that the fixed point set $\mathbb{L}^{h}$ is a connected closed subset of $\mathbb{L}$. Since connected subsets of $\mathbb{L}$ are intervals, it follows that $\mathbb{R}^{h}$ is either a point or a nontrivial interval.

If $\mathbb{L}^{h}$ is a nontrivial interval then $h$ maps $X=\mathbb{L} \backslash \mathbb{L}^{h}$ to itself. Note that $X$ is either connected or has two components.

If $X$ is connected then $h \mid X \in \mathscr{H}(X) \approx \mathscr{H}\left(\mathbb{L}_{+}\right)$, which, by Theorem 3.2, has no torsion. Thus $h \mid X$ is the identity, so that $h=e$, a contradiction.

If $X$ is disconnected then $X$ has two components, say $A$ and $B$, each of which is homeomorphic to $\mathbb{L}_{+}$. Continuity of $h$ at the end points of $\mathbb{L}^{h}$ ensures that $h(A)=A$ and $h(B)=B$, and, as in the previous paragraph, we conclude that $h=e$, a contradiction.

It follows that $\mathbb{R}^{h}$ is a single point. Hence $h(A)=B$ and $h(B)=A$, where $A$ and $B$ are the two components of $\mathbb{L} \backslash \mathbb{R}^{h}$. Thus $h^{2}(A)=A$, and, as above, we conclude that $h^{2}=e$.

LEMMA 3.4. Let $h: \mathbb{L}^{n} \rightarrow \mathbb{R}^{n}$ be a homeomorphism. Set $D=\left\{\alpha \in \omega_{1} \mid h\left(S_{\alpha}\right)=S_{\alpha}\right\}$. Then $D$ is closed and unbounded.

PROOF. $D$ is closed. Suppose that $\left\langle\alpha_{n}\right\rangle$ is a sequence in $D$ converging upwards to $\alpha$. If $y \in \stackrel{\circ}{\alpha}_{\alpha}$, then there is $n \in \omega$ such that $y \in S_{\alpha_{n}}$ so $h(y) \in h\left(S_{\alpha_{n}}\right)=S_{\alpha_{n}} \subset S_{\alpha}$. This shows that $h\left(\stackrel{\circ}{S}_{\alpha}\right) \subset S_{\alpha}$ so that $h\left(S_{\alpha}\right) \subset S_{\alpha}$. On the other hand, if $h(y) \in \stackrel{\circ}{\alpha}_{\alpha}$ then there is $n \in \omega$ such that $h(y) \in S_{\alpha_{n}}=h\left(S_{\alpha_{n}}\right)$ so $y \in S_{\alpha_{n}} \subset S_{\alpha}$ and as before $S_{\alpha} \subset h\left(S_{\alpha}\right)$. Thus $h\left(S_{\alpha}\right)=S_{\alpha}$ and hence $\alpha \in D$.

$D$ is unbounded. Suppose that $\beta \in \omega_{1}$ and set $\alpha_{0}=\beta$. Suppose that $\alpha_{n}$ has been constructed. Because $h\left(S_{\alpha_{n}}\right) \cup h^{-1}\left(S_{\alpha_{n}}\right)$ is compact, we may choose $\alpha_{n+1}>\alpha_{n}$ so that $h\left(S_{\alpha_{n}}\right) \cup h^{-1}\left(S_{\alpha_{n}}\right) \subset S_{\alpha_{n+1}}$. Suppose that $\alpha_{n} \nearrow \alpha$.

We claim that $\alpha \in D$. Suppose that $y \in S_{\alpha}$. As $\alpha_{n} \nearrow \alpha$ it follows that there is $n$ so that $y \in S_{\alpha_{n}}$. Then $h^{-1}(y) \in S_{\alpha_{n+1}} \subset S_{\alpha}$ so $y \in h\left(S_{\alpha}\right)$, that is, $\dot{S}_{\alpha} \subset h\left(S_{\alpha}\right)$ and hence $S_{\alpha} \subset h\left(S_{\alpha}\right)$. Similarly $S_{\alpha} \subset h^{-1}\left(S_{\alpha}\right)$, that is, $h\left(S_{\alpha}\right) \subset S_{\alpha}$. Thus $S_{\alpha}=h\left(S_{\alpha}\right)$ so $\alpha \in D$.

We define the open ray in $L^{n}$ through $x$ to be

$$
R(x)=\left\{y \in L^{n} \mid \frac{\|x\|}{|x|}<\frac{y_{i}}{x_{i}}=\frac{y_{j}}{x_{j}} \text { for each } i, j \in \hat{x} \text { and } y_{i}=x_{i} \text { for each } i \in \check{x}\right\} .
$$




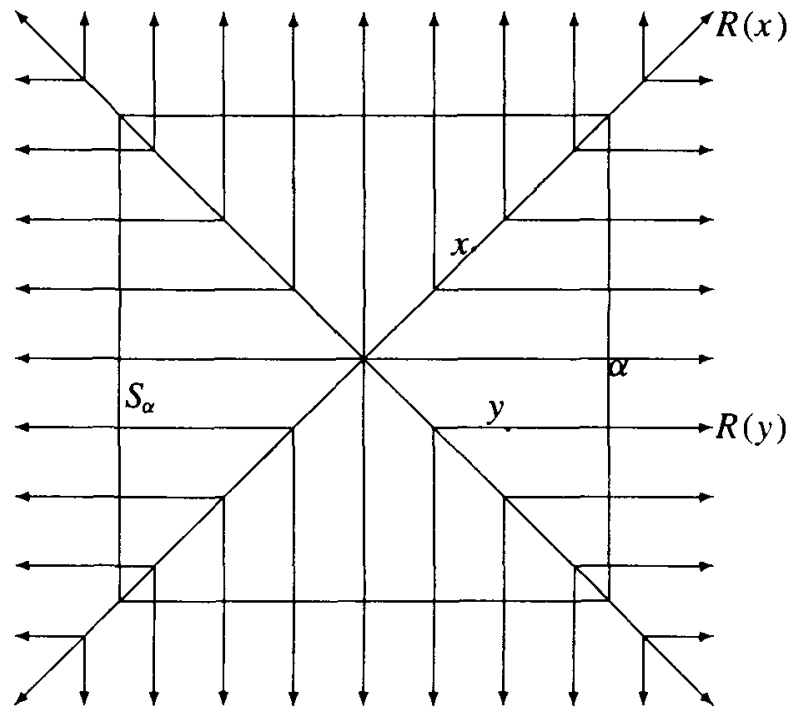

Define an equivalence relation $\sim$ on $L^{n} \backslash\{0\}$ by setting $x \sim y$ if and only if $x$ and $y$ belong to the same open ray in $L^{n} \backslash\{0\}$. Let $R_{\alpha}$ be the quotient space $\left(L^{n} \backslash S_{\alpha}\right) / \sim$ and denote the quotient map by $\pi_{\alpha}: L^{n} \backslash S_{\alpha} \rightarrow R_{\alpha}$. Then $R_{\alpha}$ consists of the ends of all the open rays. For each $\alpha, \beta \in \omega_{1}$ with $\alpha \leq \beta$ let $p_{\alpha}^{\beta}: R_{\beta} \rightarrow R_{\alpha}$ be the bijection induced by the inclusion $L^{n} \backslash S_{\beta} \subset L^{n} \backslash S_{\alpha}$. Then $p_{\alpha}^{\beta}$ is continuous (but $\left(p_{\alpha}^{\beta}\right)^{-1}$ is not unless $\beta=\alpha$ ). Thus $\left\langle p_{\alpha}^{\beta}: R_{\beta} \rightarrow R_{\alpha}\right\rangle$ is an inverse system.

DEFINITION 3.5. The ray space, $R\left(L^{n}\right)$, of $L^{n}$ is the limit of the inverse system $\left\langle p_{\alpha}^{\beta}: R_{\beta} \rightarrow R_{\alpha}\right\rangle$.

We have the following facts about $R\left(L^{n}\right)$ :

(1) There is a natural continuous bijection, which we denote by $p_{\alpha}: R\left(L^{n}\right) \rightarrow R_{\alpha}$;

(2) $R\left(L^{n}\right)$ has a natural stratification $R\left(L^{n}\right)=\bigcup_{i=0}^{n-1} R_{i}$, where each $R_{i}$ is a finite disjoint union of subsets each of which is homeomorphic to $\mathbb{L}^{i}$ when $L=\mathbb{L}$ and $\mathbb{L}_{+}^{i}$ when $L=\mathbb{L}_{+}$;

(3) $R\left(L^{n}\right)$ is a compact Hausdorff space.

$R\left(\mathbb{L}^{n}\right)$ may be thought of as the boundary of a hypercube in which each open face of dimension $i$ has been replaced by a copy of $\mathbb{L}^{i}$. Furthermore, the closure of a face of dimension $i$ is homeomorphic to the $i$ th power of the 2-point compactification of $\mathbb{L}$. For $R\left(\mathbb{L}_{+}\right)$the situation is similar except that the faces are replaced by copies of $\mathbb{L}_{+}^{i}$. One may carry out a similar construction in $\mathbb{R}^{n}$ and so get an ordinary hypercube in which each face is the 2-point compactification of $\mathbb{R}$. The following lemma shows that there is a major difference between the situations in $\mathbb{R}^{n}$ and $L^{n}$ : the analogue of this lemma in $\mathbb{R}^{n}$ is false. As a result there is a major difference between the behaviours 
of homeomorphisms of $L^{n}$, as exhibited by Corollary 3.10 , and $\mathbb{R}^{n}$.

LEMMA 3.6. If $: \omega_{1} \rightarrow L^{n}$ is an embedding then there is a unique point $r \in R\left(L^{n}\right)$ such that for each $\alpha \in \omega_{1}$ the set $e^{-1}\left(\overline{\left.\pi_{\alpha}^{-1} p_{\alpha}(r)\right)}\right.$ is a closed unbounded subset of $\omega_{1}$.

PROOF. Clearly for each $r \in R\left(L^{n}\right)$ and each $\alpha \in \omega_{1}$ we have that $e^{-1}\left(\overline{\pi_{\alpha}^{-1} p_{\alpha}(r)}\right)$ is closed so we need only show that there is a unique $r$ for which this set is unbounded. If we can show that there is at least one such $r$ then it must be unique as any two closed unbounded subsets of $\omega_{1}$ have non-empty intersection by Proposition 1.2. Thus we need only show the existence of such a point $r \in R\left(L^{n}\right)$.

Because $\omega_{1}$, and hence $e\left(\omega_{1}\right)$, is not Lindelöf whereas every bounded subset of $L^{n}$ is Lindelöf, it follows that at least 1 coordinate of $e\left(\omega_{1}\right)$ is unbounded; let us suppose that exactly $k$ coordinates of $e\left(\omega_{1}\right)$ are unbounded, and for ease of notation we will suppose that the first $k$ coordinates are unbounded. Thus

(1) for each $a \in \mathbb{L}_{+}$there is $\alpha_{a} \in \omega_{1}$ such that for each $i \leq k$ we have $\left|e_{i}\left(\alpha_{a}\right)\right| \geq a$; (2) there is $b \in \mathbb{L}_{+}$such that for each $\alpha \in \omega_{1}$ and each $i>k$ we have $\left|e_{i}(\alpha)\right| \leq b$.

Firstly we show that $E=\left\{\alpha \in \omega_{1}|| e_{i}(\alpha)|=| e_{j}(\alpha) \mid\right.$ for all $\left.i, j \leq k\right\}$ is closed and unbounded. $E$ is clearly closed, so we need only show that $E$ is unbounded. Suppose that $\beta_{0} \in \omega_{1}$. Given $\beta_{n} \in \omega_{1}$ choose $a_{n} \in \mathbb{L}_{+}$so that $e\left(\left[0, \beta_{n}\right]\right) \subset\left[-a_{n}, a_{n}\right]^{k} \times$ $[-b, b]^{n-k}$. By assumption there is $\beta_{n+1} \in \omega_{1}$ such that $\left|e_{i}\left(\beta_{n+1}\right)\right| \geq a_{n}$ for each $i \leq k$. Let $a_{n} \nearrow a$ and $\beta_{n} \nearrow \beta$. Then $\left|e_{i}(\beta)\right|=a$, so $\beta \in E$.

Now we show that there is a ray $r \in R\left(L^{n}\right)$ as described. For each $y \in \mathbb{L}^{n-k}$ consider $\mathbb{L}^{k} \times\{y\} \subset \mathbb{L}^{k} \times \mathbb{L}^{n-k}=\mathbb{L}^{n}$. It suffices to show that $e^{-1}\left(\mathbb{L}^{k} \times\{y\}\right)$ is unbounded for some $y \in[-b, b]^{n-k}$, for then this set will form a closed, unbounded subset of $\omega_{1}$, and this must intersect the closed, unbounded set $E$ of the previous paragraph in a closed, unbounded set. Suppose instead that this is not the case. Then for each $y \in[-b, b]^{n-k}$ there is $\alpha_{y} \in \omega_{1}$ such that $e^{-1}\left(\mathbb{L}^{k} \times\{y\}\right) \subset\left[0, \alpha_{y}\right)$. By continuity of $e$ it follows that $e^{-1}\left(\mathbb{L}^{k} \times\{\eta\}\right) \subset\left[0, \alpha_{y}\right)$ for each $\eta$ in some neighbourhood of $y$ in $[-b, b]^{n-k}$. Then by compactness of $[-b, b]^{n-k}$ we conclude that there is $\alpha \in \omega_{1}$ such that $e^{-1}\left(\mathbb{L}^{k} \times\{y\}\right) \subset[0, \alpha]$ for each $y \in[-b, b]^{n-k}$, which contradicts assumption (1).

LEMMA 3.7. Suppose that $X$ is a compact first countable space and $e: \omega_{1} \times X \rightarrow$ $L^{n}$ is an embedding. Then the induced map $\bar{e}: X \rightarrow R\left(L^{n}\right)$, where $\bar{e}(t)$ is that point of $R\left(L^{n}\right)$ given by Lemma 3.6 applied to $e_{t}$, is also an embedding.

Proof. To show that $\bar{e}$ is continuous we must show that for each $\alpha \in \omega_{1}$ the composition $\bar{e}_{\alpha}: X \stackrel{\bar{e}}{\longrightarrow} R\left(L^{n}\right) \stackrel{p_{\alpha}}{\longrightarrow} R_{\alpha}$ is continuous. Suppose that $t \in X$. Then $\pi_{\alpha}^{-1} \bar{e}_{\alpha}(t)$ will consist of a homeomorph of the open long ray in which some coordinates are fixed and up to sign the remaining coordinates are equal and range through $\left(\alpha, \omega_{1}\right)$ : 
without loss of generality we will assume that the first $k$ coordinates are equal and range through $\left(\alpha, \omega_{1}\right)$ and the remaining coordinates are all non-negative, so that $\pi_{\alpha}^{-1} \bar{e}_{\alpha}(t)$ is of the form $\left\{\left(x, x, \ldots, x, b_{1}, \ldots, b_{n-k}\right) \mid x>\alpha\right\}$. Then a basic neighbourhood of $\bar{e}_{\alpha}(t)$ may be taken in the form $\pi_{\alpha}(N)$, where $N=\left(\alpha, \omega_{1}\right)^{k} \times \prod_{i=1}^{n-k}\left(b_{i}^{-}, b_{i}^{+}\right)$and $b_{i}^{-}<b_{i}<b_{i}^{+}$.

Suppose that $\bar{e}_{\alpha}^{-1} \pi_{\alpha}(N)$ is not a neighbourhood of $t$. Then there is a sequence $\left\langle t_{i}\right\rangle$ of points of $X \backslash \bar{e}^{-1} \pi_{\alpha}(N)$ converging to $t$. Thus there are closed, unbounded subsets $\left\langle C_{i}\right\rangle$ and $C$ such that $e\left(C_{i} \times\left\{t_{i}\right\}\right) \cap N=\varnothing$ while $e(C \times\{t\}) \subset N$. As the countably many sets $\{C\} \cup\left\{C_{i} \mid i \in \omega\right\}$ are all closed and unbounded, by Proposition 1.2 so is their intersection; choose $\beta \in C \cap\left(\bigcap_{i \epsilon \omega} C_{i}\right)$. Then $\left\langle\left(\beta, t_{i}\right)\right\rangle$ converges to $(\beta, t)$ but $\left\langle e\left(\beta, t_{i}\right)\right\rangle$ does not converge to $e(\beta, t)$, contradicting continuity of $e$.

Now $e$ must be an injection, for if not then there will be two points $s, t \in X$ so that $\bar{e}(s)=\bar{e}(t)$. Hence there are closed, unbounded subsets $C_{s}, C_{t} \subset \omega_{1}$ so that $e_{s}\left(C_{s}\right)$ and $e_{t}\left(C_{t}\right)$ are mapped to the same ray. Then we can find two distinct points $\left(s^{\prime}, s\right),\left(t^{\prime}, t\right) \in \omega_{1} \times X$ so that $e\left(s^{\prime}, s\right)=e\left(t^{\prime}, t\right)$, contrary to $e$ being an embedding. As a continuous injection from a compact space to a Hausdorff space, $e$ is then an embedding.

LEMMA 3.8. If $f: I \rightarrow R\left(L^{n}\right)$ is a path, then $f(I) \subset R_{i}$ for some stratum $R_{i}$.

PROOF. As noted, each component of the $i$ th stratum of $R\left(L^{n}\right)$ is homeomorphic to $\mathbb{L}^{i}$ or $\mathbb{L}_{+}^{i}$, and the closure of this component is homeomorphic to the $i$ th power of the 2- or 1-point compactification. The compactification has 3 or 2 path components, viz $\mathbb{L}$ or $\mathbb{L}_{+}$and each of the extra points. Hence the closure of the component consists of $3^{i}$ or $2^{i}$ path components, one of which is the component itself. Thus $f(I)$ must lie in one of these path components, which is a subset of some stratum.

COROLlaRY 3.9. If $f: X \rightarrow R\left(L^{n}\right)$ is continuous and $X$ is path connected, then $f(X) \subset R_{i}$ for some stratum $R_{i}$.

COROLLARY 3.10. Any homeomorphism $h: L^{n} \rightarrow L^{n}$ induces a homeomorphism $\bar{h}: R\left(L^{n}\right) \rightarrow R\left(L^{n}\right)$. Moreover, $\bar{h}\left(R_{i}\right)=R_{i}$ for each stratum $R_{i}$.

PROOF. Given $r \in R\left(L^{n}\right)$ we apply Lemma 3.6 to $\pi_{\alpha}^{-1} p_{\alpha}(r)$, which contains a subset order equivalent to $\omega_{1}$, to find the natural candidate for $\bar{h}(r)$. By Corollary 3.9 $\bar{h}$ takes each component of a stratum to a stratum and by Lemma 3.7 it embeds each such stratum; hence no stratum is taken by $\bar{h}$ to a stratum of lower dimension. Applying the same reasoning to $h^{-1}$ we conclude that $\bar{h}$ carries a stratum of dimension $i$ to one of the same dimension. Continuity of $\bar{h}$ and its inverse is similar to the proof of continuity in Lemma 3.7. 
Let $\mathscr{G}_{n}$ be the group of symmetries of the hypercube $[-1,1]^{n}$ and $\mathscr{G}_{+. n}$ be the group of symmetries of the hypercube $[0,1]^{n}$ which send any point with at least one coordinate equal to 0 to another such point and any point with no coordinate equal to 0 to another such point. Let $\mu: \mathscr{G}_{n} \rightarrow \mathscr{H}\left(\mathbb{L}^{n}\right)$ and $\mu_{+}: \mathscr{G}_{+, n} \rightarrow \mathscr{H}\left(\mathbb{L}_{+}^{n}\right)$ be the natural monomorphisms. By Corollary 3.10 there are homomorphisms $\varphi$ : $\mathscr{H}\left(\mathbb{L}^{n}\right) \rightarrow \mathscr{H}\left(R\left(\mathbb{L}^{n}\right)\right)$ and $\varphi_{+}: \mathscr{H}\left(\mathbb{L}_{+}^{n}\right) \rightarrow \mathscr{H}\left(R\left(\mathbb{L}_{+}^{n}\right)\right)$. The compositions $\varphi \mu:$ $\mathscr{G}_{n} \rightarrow \mathscr{H}\left(R\left(\mathbb{L}^{n}\right)\right)$ and $\varphi_{+} \mu_{+}: \mathscr{G}_{+, n} \rightarrow \mathscr{H}\left(R\left(\mathbb{L}_{+}^{n}\right)\right)$ are also monomorphisms.

THEOREM 3.11. Suppose that $h \in \mathscr{H}\left(\mathbb{L}_{+}^{n}\right)$ is an element of finite order $q$.

(1) If $n=1$, then $q=1$.

(2) If $n=2$, then $q=1$ or 2 .

(3) If $n=3$, then $q=1,2$ or 3 .

(4) If $n=4$, then $q=1,2,3,4$ or 6 .

ProOF. For any $\alpha \in \omega_{1}$ set

$$
\begin{aligned}
V_{\alpha}=\left\{\left(x_{1}, \ldots, x_{n}\right) \in \mathbb{L}_{+}^{n} \mid x_{i}\right. & =0 \text { for exactly one coordinate } i \text { and } \\
x_{i} & =\alpha \text { for all others }\} .
\end{aligned}
$$

By Proposition 1.1, Lemma 3.4 and Corollary 3.10 there is a closed unbounded subset $A \subset \omega_{1}$ so that for each $\alpha \in A$ we have $h\left(S_{\alpha} \cap \mathbb{L}_{+}^{n}\right)=S_{\alpha} \cap \mathbb{Q}_{+}^{n}$ and $h\left(V_{\alpha}\right)=V_{\alpha}$. Fix $\alpha \in A$ : it suffices to show that the $q$ th power of $h \mid S_{\alpha} \cap \mathbb{L}_{+}^{n}$ is the identity, where $q$ is as in the theorem.

Case 1: $h$ fixes $V_{\alpha}$. If necessary replace $h$ by a power of $h$ so that $q$ is a power of some prime $p$.

Consider $h \mid \partial\left(\mathbb{S}_{\alpha} \cap \partial \mathbb{Q}_{+}^{n}\right)$ when $n \geq 3$ : because $\partial\left(\mathbb{S}_{\alpha} \cap \partial \mathbb{Q}_{+}^{n}\right) \approx \mathbb{S}^{n-2}$ and $h$ fixes $n$ points therein, it follows from the P. A. Smith theorem [2, Theorem III.5.1] that the fixed point set of $h \mid \partial\left(\mathbb{S}_{\alpha} \cap \partial \mathbb{Q}_{+}^{n}\right)$ is a mod $p$ homology $r$-sphere, where $n-2-r$ is even if $p$ is odd and $h$ is an orientation-reversing involution if $p=2$. If $n=3$, then $\partial\left(\mathbb{S}_{\alpha} \cap \partial \mathbb{L}_{+}^{n}\right) \approx \mathbb{S}^{1}$ so that $h$ must fix all of $\partial\left(\mathbb{S}_{\alpha} \cap \partial \mathbb{L}_{+}^{n}\right)$. If $n=4$, then $\partial\left(\mathbb{S}_{\alpha} \cap \partial \mathbb{L}_{+}^{n}\right) \approx \mathbb{S}^{2}$ and $h$ has at least 4 fixed points on this set. Furthermore if $p$ is odd, then the only way for $n-2-r$ to be even is for $r=0$ or 2 . Now it is not possible to have $r=0$ by the P. A. Smith theorem [2, Theorem III.5.2] because there are more than 2 fixed points. On the other hand, $r=2$ means that $h$ fixes all of $\partial\left(\mathbb{S}_{\alpha} \cap \partial \mathbb{L}_{+}^{n}\right)$. Thus when $n \leq 3, h$ fixes $\partial\left(\mathbb{S}_{\alpha} \cap \partial \mathbb{R}_{+}^{n}\right)$ and even when $n=4, h$ either fixes $\partial\left(\mathbb{S}_{\alpha} \cap \partial \mathbb{L}_{+}^{n}\right)$ or is an involution there, that is, $h^{2}$ fixes $\partial\left(\mathbb{S}_{\alpha} \cap \partial \mathbb{L}_{+}^{n}\right)$.

Firstly consider the case where $h$ fixes $\partial\left(\mathbb{S}_{\alpha} \cap \partial \mathbb{L}_{+}^{n}\right)(n \geq 2)$. Consider $h \mid \mathbb{S}_{\alpha} \cap \partial \mathbb{L}_{+}^{n}$. As $\mathbb{S}_{\alpha} \cap \partial \mathbb{L}_{+}^{n} \approx \mathbb{B}^{n-1}$ and $h$ fixes the boundary of this set it follows from the P. A. Smith theorem [2, Theorem III.5.2] that $h$ fixes all of $\mathbb{S}_{\alpha} \cap \partial \mathbb{L}_{+}^{n}$. Similarly $h$ fixes all of $\overline{\partial\left(\mathbb{S}_{\alpha} \cap \mathbb{L}_{+}^{n}\right) \backslash \partial \mathbb{L}_{+}^{n}}$. Thus $h$ fixes all of $\partial\left(S_{\alpha} \cap \mathbb{L}_{+}^{n}\right)$, and this also holds if $n=1$. 
Applying [2, Theorem III.5.2] once again, it follows that $h$ fixes all of $S_{\alpha}$, that is, is the identity there as claimed.

Secondly consider the case where $h$ is an involution and apply the argument to $h^{2}$ to conclude that $h^{2}$ is the identity.

General Case. As $h\left(V_{\alpha}\right)=V_{\alpha}$, it follows that some power of $h$ fixes $V_{\alpha}$, and this power must be 1 when $n=1$, it must be 1 or 2 when $n=2$, it must be 1,2 or 3 when $n=3$ and it must be $1,2,3$ or 4 when $n=4$; call it $q$. Thus we may apply Case 1 to $h^{q}$ to conclude that either $h^{q}$ or $h^{2 q}$ is the identity. However when $n=4$ the involution subcase does not arise when $q=2$ or 4 as then $h^{q}$ is already orientation-preserving.

THEOREM 3.12. For $n=1,2$ and for any element of $\mathscr{H}\left(\mathbb{L}^{n}\right)$ of finite order there is an element of $\mathscr{G}_{n}$ having the same order.

Proof. Suppose that $h: \mathbb{L}^{n} \rightarrow \mathbb{L}^{n}$ is a homeomorphism of finite order. Then the corresponding homeomorphism $\bar{h}$ from Corollary 3.10 must map the strata of $R\left(\mathbb{L}^{n}\right)$ to themselves. Furthermore if two points $r, s \in R_{0}$ are such that $\pi_{\alpha}^{-1}(s)$ is obtained from $\pi_{\alpha}^{-1}(r)$ by changing the signs on each coordinate (so they are on opposite ends of a diagonal when $n=2$ ) then the same applies to their images. It follows that there is an element $\gamma \in \mathscr{G}_{n}$ such that $\varphi \mu(\gamma)\left|R_{0}=\bar{h}\right| R_{0}$.

Suppose that $\gamma$ has order $l$. We claim that $h$ also has order $l$. By Lemma 3.4, Lemma 3.6, Corollary 3.10 and Proposition 1.1 there is a closed, unbounded subset of $\omega_{1}$ such that for each $\alpha$ in this set we have $h\left(S_{\alpha}\right)=S_{\alpha}$ and $h \pi_{0}^{-1} p_{0}\left(R_{i}\right) \subset \pi_{0}^{-1} p_{0}\left(R_{i}\right)$ for each stratum $R_{i}$. It suffices to show that $h^{\prime} \mid S_{\alpha}$ is the identity. We firstly show that $h^{l} \mid \partial S_{\alpha}$ is the identity.

The set $\partial S_{\alpha} \cap \pi_{0}^{-1} p_{0}\left(R_{0}\right)$ consists of isolated points, the ends of the interval or the vertices of the square, so by choice of $\gamma$, it follows that $h^{l}$ fixes these points. Thus $h^{l}$ is the identity on $\partial S_{\alpha}$ for the case $n=1$. For the case $n=2$, because $h^{l}$ sends $\partial S_{\alpha}$ to itself, fixes the corners and is of finite order, again it follows that $h^{l}$ is the identity on $\partial S_{\alpha}$.

Now consider $h^{l} \mid S_{\alpha}$. Because it is the identity on the boundary and has finite order, and $S_{\alpha}$ is homeomorphic to the unit ball in $\mathbb{R}^{n}$, it follows from [2, Theorem III 5.2] that $h^{l} \mid S_{\alpha}$ is the identity.

EXAMPLE 3.13. It is not the case that the only homeomorphisms of $\mathbb{L}^{2}$ of finite order are the eight which possibly interchange the coordinates and possibly reverse the direction of one or both.

For example let $g: \mathbb{L}_{+} \times \mathbb{L} \rightarrow \mathbb{L}_{-} \times \mathbb{L}$ be a homeomorphism which sends $(x, y)$ to $(-x, y)$ on all except a non-empty open set contained in $\mathbb{L}_{0} \times \mathbb{L}$. Let $h: \mathbb{L}^{2} \rightarrow \mathbb{L}^{2}$ be $g$ on $\mathbb{L}_{+} \times \mathbb{L}$ and $g^{-1}$ on $\mathbb{L}_{-} \times \mathbb{L}$. Then $h$ has order 2 . 
QUESTION 3.14. Can we somehow be more specific concerning the homeomorphisms of finite order?

For example are they all isotopic to a homeomorphism which permutes and possibly reverses coordinates?

\section{QUESTION 3.15. Do Theorem 3.11 and Theorem 3.12 hold for all $n$ ?}

We should be more specific with respect to Theorem 3.11: the intention of the question is that the torsion in $\mathscr{H}\left(\mathbb{L}_{+}^{n}\right)$ should be the same as that of $\mathscr{G}_{+, n}$. As the statement of Theorem 3.11 part 4 does not satisfy this condition, the question includes the case where $n=4$.

Certainly large parts of the proofs are valid for the general case. The generalisation of the proof of Theorem 3.12 breaks down at the point where we show that $h^{l} \mid \partial S_{\alpha}$ is the identity because we cannot be sure that $h$ sends each $i$-face of $\partial S_{\alpha}$ to an $i$-face except for $i=0$. We have been able to show that for $\alpha$ in some closed, unbounded set there is a subset of each $i$-face which has non-empty interior in the face and which is mapped by $h$ to an $i$-face but this does not appear to be sufficient to complete the proof.

\section{Acknowledgement}

The authors thank the referee for a number of valuable suggestions which helped improve the exposition.

\section{References}

[1] A. Borel, Seminar on transformation groups, Ann. of Math. Stud. 46 (Princeton University Press, Princeton, 1960).

[2] G. E. Bredon, Introduction to compact transformation groups (Academic Press, New York, 1972).

[3] - Sheaf theory, 2nd edition (Springer, New York, 1997).

[4] D. Gauld, 'Homeomorphisms of 1-manifolds and $\omega$-bounded 2-manifolds', in: Papers on general topology and applications (Ann. New York Acad. Sci., New York, 1993) pp. 142-149.

[5] K. Kunen, Set theory, an introduction to independence proofs (North Holland, Amsterdam, 1980).

[6] E. H. Spanier, Algebraic topology (McGraw Hill, New York, 1966).

Department of Mathematics

R. D. University

Jabalpur

(M. P.) 482001

India
Department of Mathematics The University of Auckland Private Bag 92019 Auckland New Zealand 